\title{
Negative effect of dietary elimination on breastfeeding mothers having infants with and without food allergy
}

\author{
OZLEM YILMAZ1 ${ }^{1}$, Anil Safak Kacar ${ }^{1}$, Emre Gogebakan ${ }^{1}$, Ceren $_{\text {Can }}^{2}$, Isil Necef ${ }^{1}$, Tuba \\ Mutluer $^{1}$, Nuray Uslu Kızılkan ${ }^{1}$, Ali Sarper Taskiran ${ }^{1}$, and Cansin Sackesen ${ }^{1}$ \\ ${ }^{1}$ Koc University School of Medicine \\ ${ }^{2}$ Istanbul Bakirkoy Dr Sadi Konuk Training and Research Hospital
}

May 3, 2021

\begin{abstract}
Abstract Background: There has been no trial evaluating the psychopathology in breastfeeding mothers of infants with food allergy (FA). Objective: To investigate the effect of dietary elimination on maternal psychopathology, specifically stress/anxiety and mother-to-infant bonding and explore the importance of sociodemographic features on these variables. Methods: Breastfeeding mothers following an elimination diet due to FA in their children aged 1-to-12 months were compared with the healthy controls. Physician-diagnosed FA group were divided into IgE-, non-IgE-mediated and infants with some minor symptoms which were not enough to make the diagnosis of FA were classified as Indecisive symptoms for FA group. Mothers completed standardized questionnaires including Symptom Checklist 90R, Beck Depression/Anxiety Inventories (BDI/BAI), Postpartum Bonding Questionnaire (Bonding). Results: Of 179 mother-infants, 64 were healthy, 89 were FA, 16 were indecisive symptoms for FA. The mean age of the mothers and infants were $31.1 \pm 4.7$ years and $6.3 \pm 3.6$ months. The physician diagnosed FA groups had higher scores for anxiety $(\mathrm{p}=0.008)$, anger $(\mathrm{p}=0.042)$, depression $(\mathrm{p}<0.001)$, obsession $(\mathrm{p}=0.002)$, phobia $(\mathrm{p}=0.008)$, somatization $(\mathrm{p}=0.002)$ and general symptom index (GSI) $(\mathrm{p}=0.001)$, BDI $(\mathrm{p}<0.001)$, BAI $(\mathrm{p}=0.008)$ and Bonding [attachment $(\mathrm{p}=0.001)$, anger $(\mathrm{p}=0.019)$ and total $(\mathrm{p}=0.036)]$ than the healthy. The indecisive symptoms for FA group had a similar score pattern to physician-diagnosed FA except interpersonal sensitivity, BDI and attachment. Conclusion: Breastfeeding mothers of infants with FA were anxious, depressive and had many psychopathologies which affected bonding. Interventions targeting negativity in caregivers' social relationships are urgently needed.
\end{abstract}

\section{Negative effect of dietary elimination on breastfeeding mothers having infants with and without food allergy}

Ozlem Yilmaz ${ }^{1}$, Anıl Safak Kacar ${ }^{2}$, Emre Gogebakan $^{3}$, Ceren $\mathrm{Can}^{4}$, Isil Necef ${ }^{5}$, Tuba Mutluer ${ }^{5}$, Nuray Uslu Kizilkan $^{6}$, Ali Sarper Taskiran ${ }^{5,7}$, Cansin Sackesen ${ }^{1}$

${ }^{1}$ Koc University, School of Medicine, Division of Pediatric Allergy, Istanbul

${ }^{2}$ Koc University, Graduate School of Health Sciences, Koc University Research Center for Translational Medicine, Istanbul

${ }^{3}$ Koc University, School of Medicine, İstanbul

${ }^{4}$ Bakirkoy Dr. Sadi Konuk Education and Research Hospital, Pediatric Allergy and Immunology Clinic, Istanbul

${ }^{5}$ Koc University, School of Medicine, Department of Child and Adolescent Psychiatry, Istanbul

${ }^{6}$ Koc University, School of Medicine, Division of Pediatric Gastroenterology, Istanbul, Turkey 
${ }^{7}$ Child Mind Institute, New York, USA

Corresponding Author:

Cansin Sackesen, MD, Prof. Dr.

Koc University School of Medicine, Division of Pediatric Allergy

E-mail: csackesen@ku.edu.tr; csackesen@yahoo.com

Davutpasa Street No: 4, Topkapi 34010 /Istanbul/Turkey

Ozlem Yilmaz, MD, Assoc. Prof.

Koc University School of Medicine, Division of Pediatric Allergy

e-mail: drozlemyilmaz09@gmail.com

Anil Safak Kacar, MD

Koc University Graduate School of Health Sciences, Koc University Research Center for Translational Medicine, Istanbul

e-mail: akacar@ku.edu.tr

Emre Gogebakan,

Koc University School of Medicine, İstanbul

e-mail: egogebakan15@ku.edu.tr

Ceren Can, MD

Bakirkoy Dr. Sadi Konuk Education and Research Hospital, Pediatric Allergy and Immunology Clinic, Istanbul

e-mail: cereni35@yahoo.com

\section{Isil Necef, MA}

Koc University School of Medicine, Department of Child and Adolescent Psychiatry, Istanbul e-mail: isilnecef@gmail.com

Tuba Mutluer, MD

Koc University School of Medicine, Department of Child and Adolescent Psychiatry, Istanbul e-mail: tmutluer@kuh.ku.edu.tr

Nuray Uslu Kizilkan, Assoc. Prof.

Koc University School of Medicine, Division of Pediatric Gastroenterology, Istanbul, Turkey e-mail: nurayu74@yahoo.com

Ali Sarper Taskiran, MD

Koc University School of Medicine, Department of Child and Adolescent Psychiatry, Istanbul

Child Mind Institute, New York

e-mail:sarper.taskiran@childmind.org

Cansin Sackesen, MD, Prof. Dr.

Koc University School of Medicine, Division of Pediatric Allergy 
E-mail: csackesen@ku.edu.tr; csackesen@yahoo.com

There is no funding for the study.

Word count for the abstract: 234

Word count for the article: 2994

Highlights box:

Children, adolescents and adults with FA and their families were observed to have low quality of life. This trial evaluated t

Key Words: Anxiety, breastfeeding, bonding, elimination diet, food allergy, IgE-mediated, Non-IgE mediated, psychopathology, stress

\section{Abbreviations:}

BAI: Beck Anxiety Inventory

BDI: Beck Depression Inventory

Bonding: Brockington Postpartum Bonding Questionnaire

FA: Food allergy

False-FA: False food allergy

IgE: IgE-mediated

Non-IgE: Non-IgE-mediated

QoL: Quality of life

True-FA: True food allergy

SCL-90R: Symptom Checklist 90

Negative effect of dietary elimination on breastfeeding mothers having infants with and without food allergy

\section{Abstract}

Background: There has been no trial evaluating the psychopathology in breastfeeding mothers of infants with food allergy (FA).

Objective: To investigate the effect of dietary elimination on maternal psychopathology, specifically stress/anxiety and mother-to-infant bonding and explore the importance of sociodemographic features on these variables.

Methods: Breastfeeding mothers following an elimination diet due to FA in their children aged 1-to-12 months were compared with the healthy controls. Physician-diagnosed FA group were divided into IgE-, non-IgE-mediated and infants with some minor symptoms which were not enough to make the diagnosis of FA were classified as Indecisive symptoms for FA group. Mothers completed standardized questionnaires including Symptom Checklist 90R, Beck Depression/Anxiety Inventories (BDI/BAI), Postpartum Bonding Questionnaire (Bonding).

Results: Of 179 mother-infants, 64 were healthy, 89 were FA, 16 were indecisive symptoms for FA. The mean age of the mothers and infants were $31.1 \pm 4.7$ years and $6.3 \pm 3.6$ months. The physician diagnosed FA groups had higher scores for anxiety $(\mathrm{p}=0.008)$, anger $(\mathrm{p}=0.042)$, depression $(\mathrm{p}<0.001)$, obsession $(\mathrm{p}=0.002)$, phobia $(\mathrm{p}=0.008)$, somatization $(\mathrm{p}=0.002)$ and general symptom index (GSI) $(\mathrm{p}=0.001)$, BDI $(\mathrm{p}<0.001)$, BAI $(\mathrm{p}=0.008)$ and Bonding [attachment $(\mathrm{p}=0.001)$, anger $(\mathrm{p}=0.019)$ and total $(\mathrm{p}=0.036)]$ than the healthy. The 
indecisive symptoms for FA group had a similar score pattern to physician-diagnosed FA except interpersonal sensitivity, BDI and attachment.

Conclusion: Breastfeeding mothers of infants with FA were anxious, depressive and had many psychopathologies which affected bonding. Interventions targeting negativity in caregivers' social relationships are urgently needed.

\section{Negative effect of dietary elimination on breastfeeding mothers having infants with and without food allergy}

\section{Introduction}

Evidence indicates that the prevalence of food allergy (FA) has increased in the last decade (1). However, the pathogenesis of FA has not completely been understood, yet. There is currently no cure or preventive treatment for FA (2). Previous studies showed that children, adolescents and adults with FA and their families had low quality of life (QoL) (3-5). However, the QoL in infants was investigated scarcely and there has been no trial evaluating the psychosocial well-being in breastfeeding mothers having infants with FA (6).

The first year of life is unique to the rest of life. There can be several factors that may impact QoL in motherinfant dyads (7). Many breastfeeding mothers believe that foods transferring through the breast milk from mother to infant can lead to allergy. Due to this perspective, many breastfeeding mothers who think that their infants had FA lean to remove many nutrients from their diets. The diagnosis and suspicion of FA and dietary elimination may cause stress, anxiety in and affect psychosocial well-being of mothers.

The aim of this study was to investigate the effect of dietary elimination on maternal psychopathology among mothers, specifically stress/anxiety and mother-to-infant bonding. Our secondary aim was to explore the importance of sociodemographic features on these variables in this critical period of mother-to-infant bonding in order to provide specific psychosocial approaches. The assessment of psychosocial status of mothers in the first years of their children's life may be important to understand maternal stress and to maintain a new approach and behavior to the mothers who are anxious.

\section{Methods}

\section{Study population}

The study population was collected from three Pediatric allergy (Koc University, Mersin City Hospital, Bakirkoy Dr. Sadi Konuk Research and Training Hospital) and one Pediatric Gastroenterology (Koc University Pediatric Gastroenterology) centers from Turkey. The study population consisted of breastfeeding mothers who were on an elimination diet due to a diagnosis of FA in their children aged 1-to-12 months. The breastfeeding mothers presented to the study centers who were willing to participate in the study were included, consecutively. Food allergy was classified as $\operatorname{IgE}$-mediated ( $\operatorname{IgE}$ ), non-IgE-mediated (Non-IgE) and mixed (both IgE- and non-IgE-mediated FA) FA. The diagnosis of IgE-mediated FA was established according to the following criteria: 1. A consistent and clear-cut history of FA-related symptoms that developed within minutes to hours following the ingestion of food, 2. Positive serum levels of $\operatorname{sIgE}(>0.35 \mathrm{kU}$ A $/ \mathrm{L})$ for the specific food, 3. Positive skin prick test (SPT) for the specific food (a wheal diameter of $3 \mathrm{~mm}$ or greater than the negative control). The diagnosis of non-IgE mediated FA was established according to the following criteria: 1 . The presence of blood in the stool after in otherwise healthy infants and the disappearance of blood with the elimination of the suspected food(s) from maternal diet within 1 week, 2 . The presence of isolated gastrointestinal symptoms, such as repetitive-to-profuse emesis and vomiting with diarrhea, which can lead to dehydration, lethargy, hypotension, or shock, 1-3 hours after the ingestion of the suspected food or with persistence of these symptoms for 2 weeks without any other cause and also with resolution of symptoms after the removal of the causative food(s) from the diet, with a recurrence of symptoms when reintroducing the causative food(s). The mothers on dietary elimination whose infants had a diagnosis of FA according to the criteria specified above were called as FA.

The mothers following an elimination diet with the thought of FA in their children despite the absence of 
diagnostic criteria and laboratory findings consistent with FA in their children were called as "Indecisive symptoms for food allergy". The considerations regarded as Indecisive symptoms for food allergy were as follows: 1. Mucous but no blood in stool, 2. Colic, sleep problems, irritability but no vomiting, diarrhea, loss of weight and percentiles for height \& weight were in normal range and gain of weight were within normal limits.

The control group involved breastfeeding mothers of healthy children at the same age range having no allergic, chronic or systemic disease who were admitted to well-child care outpatient clinic at the study centers. This group was called as "Healthy" or "Healthy controls" throughout the study. The Koc University Ethics Committee approved the study and written informed consent were obtained from all participants.

\section{Tools}

The participants were asked to self-complete a standardized questionnaire during their routine outpatient clinic visits. The standardized questionnaire consisted of sociodemographic features including age, number of siblings, primary and other caregivers of the child, sociodemographic features of the mother, history of any allergic, psychiatric or physical disease in the mother/family, any medications used for psychiatric disease, detailed dietary history including number and categories of foods eliminated from the mother's diet, source of information for dietary recommendations, (physician/written sources/internet/social environment/friends/relatives). Any weight loss in the mother, change in volume, consistency or cessation of breast milk, any changes in feeding frequency of infant and validated tools assessing the psychosocial functioning of the mother were also collected.

The validated tools were the following:

1. Symptom Checklist 90 (SCL-90R): SCL-90R is a self-administered psychiatric symptom checklist consisting of 90 items on a likert scale ranged from 0-to-9. SCL-90R was developed by Derogatis et al (8). and validated in Turkish by Dag et al (9).

2. Beck Depression Inventory (BDI): BDI which evaluated the risk and severity of depression in adults with a cut-off point of 17 points was developed in 1961 by Beck et al. (10) and validated in Turkish by Hisli et al. in 1989 (11).

3. Beck Anxiety Inventory (BAI): BAI assesses the frequency of anxiety symptoms composed of 21 items on a likert scale ranged from 0-to-3. BAI developed in 1998 by Beck et al (12) and validated in Turkish by Ulusoy et al in 1998 (13).

4. Brockington Postpartum Bonding Questionnaire (Bonding):Bonding was developed to evaluate the problems of mother-to-infant relationship on a 6-item likert scale described as "every day", "very often", "often", "sometimes", "rare", "never" pointed 0-5. The questionnaire composed of 25-item, 17 of which were inversely associated and validated (14-15). The scale consists of four subscales such as the General factor (12 items), Rejection and Pathological Anger (7 items), Infant-focused Anxiety (4 items) and Incipient Abuse (2 items). The cut-off scores are General factor [?] 12, Rejection and Pathological Anger [?] 17, Infant-focused Anxiety [?] 10 and Incipient Abuse [?] 3, respectively.

\section{Statistical Analysis}

Statistical analysis was performed using the Statistical Package for the Social Sciences (SPSS) version 21.0 software statistical package program (SPSS Inc., Chicago, IL). The variables were investigated to determine whether or not they are normally distributed. Descriptive analyses were presented using means and standard deviations for normally distributed values and frequencies were presented as percentages. Mean and median values of two-independent groups compared with independent samples t-test or Mann Whitney U test where appropriate. Multiple groups were compared with Kruskal Wallis test and The Jonckheere-Terpstra test was used to determine if there was a statistically significant trend across groups. The Chi-square or Fisher's exact test, where appropriate, was used to compare proportions in different groups. A p-value of less than 0.05 was considered to show a statistically significant result. 


\section{Results}

One hundred and sixty-nine mothers-infant dyads were recruited in the study between January 2016 and August 2017. The mean age of the mothers and the infants were $31.1+-4.7$ years and $6.3+-3.6$ months. Of the infants, $48.6 \%$ were female.

Of the participants, 64 were healthy controls, 89 were FA, 16 were Indecisive symptoms for FA. In the diagnosed FA group $84.2 \%, 11.2 \%$ and $4.5 \%$ were Non-IgE-mediated FA $(\mathrm{n}=75)$, IgE-mediated FA $(\mathrm{n}=10)$ and mixed FA (IgE and non-IgE-mediated FA, $\mathrm{n}=4$ ), respectively. The demographic features of the participants were presented on Table 1.

The educational level of mothers and fathers (for the mothers $\mathrm{p}=0.001$, for the fathers $\mathrm{p}=0.009$ ) was significantly higher in the Indecisive symptoms for FA group as compared to the healthy group. Being the primary caregiver as a mother was more frequently observed in Indecisive symptoms for FA group than the healthy group $(\mathrm{p}=0.006)$.

\section{Dietary characteristics in FA vs Indecisive symptoms for FA and IgE vs Non-IgE}

The mean durations of elimination diet of FA (103+-81 days) and Indecisive symptoms for FA ( $81+-59$ days) groups were similar $(\mathrm{p}=0.21)$. The most common reason to begin an elimination diet was the recommendation by a doctor. The written sources and internet were the second most common reasons for both groups (Table 2).

We asked the mothers the significance of dietary elimination in their lives. Of the mothers, $69 \%$ of FA and $82 \%$ Indecisive symptoms for FA groups reported that the eliminated foods were very important for them and avoidance of many foods affected their lives very seriously. In the FA and Indecisive symptoms for FA groups, $77 \%$ and $67 \%$ of the mothers reported that they lost weight after elimination diet. Of the mothers, $65 \%$ of FA and $57 \%$ of Indecisive symptoms for FA embodied a change in their breast milk characteristics. The decrease in breast milk volume and the increase in feeding frequency were the most frequent changes reported by the mothers in both groups (Table 2).

Egg, milk, red meat and tree nuts which were the main source of protein and calcium were the most common foods eliminated from mothers' diets. Of the groups, $41 \%$ of the FA and $29 \%$ of the Indecisive symptoms for FA excluded $>20$ nutrients from their diets. The number of foods eliminated from diets of mothers was insignificant between the groups of FA vs Indecisive symptoms for FA and IgE vs non-IgE mediated FA $(\mathrm{p}>0.05)$ (Table 3). The number of eliminated foods were presented for each group in Table 4.

\section{Comparisons among mothers of the Healthy, FA and Indecisive symptoms for FA groups}

Psychosocial functioning of the mothers was evaluated by SCL90R, BDI, BAI and Bonding.

Most of the scores for psychosomatic symptoms that were evaluated by SCL-90R namely anxiety $(\mathrm{p}=0.008)$, anger $(\mathrm{p}=0.042)$, depression $(\mathrm{p}<0.001)$, interpersonal sensitivity $(\mathrm{p}=0.001)$, obsession $(\mathrm{p}=0.002)$, phobia $(p=0.008)$, somatization $(p=0.002)$ and general symptom index (GSI) $(p=0.001)$; scores for BDI $(p=<0.001)$, BAI $(\mathrm{p}=0.008)$ and Bonding [attachment $(\mathrm{p}=0.001)$, anger $(\mathrm{p}=0.019)$ and total $(\mathrm{p}=0.036)$ ] were higher in the FA group than the healthy controls. The mothers in the Indecisive symptoms for FA had also higher scores for SCL-90R [anxiety $(\mathrm{p}=0.034)$, anger $(\mathrm{p}=0.045)$, depression $(\mathrm{p}=0.005)$, obsession $(\mathrm{p}=0.009)$, phobia $(\mathrm{p}=0.024)$, somatization $(\mathrm{p}=0.029)$ and GSI $(\mathrm{p}=0.037)]$, BAI $(\mathrm{p}=0.031)$, Bonding [anger $(\mathrm{p}=0.003)$ ] than the healthy controls. The FA and Indecisive FA groups did not differ from one another in any of the psychosocial functioning scores ( $\mathrm{p}>0.05$ for all scores) (Table 5).

Cases with FA group were categorized into IgE- and Non-IgE-mediated FA. We compared the healthy controls with the IgE- / Non-IgE-mediated and Indecisive symptoms for FA groups. The SCL-90R [anxiety $(\mathrm{p}=0.02)$, anger $(\mathrm{p}=0.005)$, depression $(\mathrm{p}=0.003)$, interpersonal sensitivity $(\mathrm{p}=0.010)$, obsession $(\mathrm{p}<0.001)$, phobia $(\mathrm{p}=0.03)$, somatization $(\mathrm{p}=0.001)$, GSI $(\mathrm{p}=0.001)]$, BDI $(\mathrm{p}=0.001)$, BAI $(\mathrm{p}=0.007)$ and Bonding [attachment $(\mathrm{p}=0.006)$ and anger $(\mathrm{p}=0.001)]$ scores were higher in Indecisive symptoms for FA than Non- 
IgE FA, and the scores of Non-IgE FA were higher than those of IgE-mediated FA (scores of Indecisive symptoms for FA > Non-IgE FA > IgE FA, p for trend is significant for each mentioned score) (Table 6).

\section{Discussion}

The present study showed that FA is negatively linked to psychosocial states of breastfeeding mothers. Psychosomatic symptoms, depression and anxiety were more frequent in breastfeeding mothers of infants with FA than healthy controls. The negative psychosocial changes were more severe in mothers with Indecisive symptoms for FA than those of mothers of infants with Diagnosed FA. The scores were also higher in nonIgE-mediated FA than those of IgE-mediated. Doctor recommendation was the most frequent reason for dietary elimination for the breastfeeding mothers.

The importance of the link between feeding and bonding have long been explored (16). It has been shown that mothers' attachment style was associated with postpartum psychological well-being and feeding preferences (17). When putting these points into consideration, it is not surprising that FA has many impacts on multiple aspects of psychological well-being. The diagnosis of FA, daily management of allergen avoidance, anxiety and fear about an unpredictable risk of allergic reactions bring a greater psychological burden to both the patients and their families (18-21). The negative impact of FA on QoL as well as emotional states such as anxiety and depression has been shown on several studies conducted on children, adolescents, adults and their families $(3,18)$. However, the impact of FA on psychosocial aspects in the first year of life was not investigated, previously.

This study evaluated FA in the first year of life which was a very sensitive and vulnerable period of mother-to infant bonding and showed that the diagnosis of FA was related to the psychosocial well-being of breastfeeding mothers, negatively. When compared with the healthy controls, the consideration of FA, irrespective of the diagnosis is FA- or Indecisive symptoms for FA caused anxiety, depression, anger, phobia, somatization symptoms, feeling of inadequacy with increased expectation of rejection and criticism. Furthermore, this study showed that FA affected mother-to-infant bonding inducing anxiety and anger in the mother and also increased interpersonal sensitivity of the mothers.

In QoL research on FA, mothers reported a significantly greater impact on psychological and physical QoL than fathers $(3,23-25)$. Various studies also showed that mothers felt the pressure of being primarily responsible for the state of health and safety of their children $(25,26)$. Therefore, it is highly likely that breastfeeding mothers may feel guilty themselves due to food allergens passing via their breast milk. When the possibility of harming their infants come together with the great responsibility of feeding, mothers may experience stress and feel helpless. They may be afraid of harming their infants and begin extensive elimination diets with great desperation. There may be such a huge intra-psychic conflict among mothers that they may develop some psychotic and somatic symptoms, which disturb mother-to-infant bonding. Dieting can also play a negative role in mother and infant relationship due to its difficulty in implementing through the daily routine for a breastfeeding mother (27).

According to a previous study, anxiety and depression of parents began even before the establishment of the FA diagnosis (28). The psychosocial scores in this study were higher in the Indecisive symptoms for FA group than in the Physician Diagnosed FA group. The Indecisive symptoms for FA group consisted of parents with higher education levels. And also, a higher ratio of the mothers in the Indecisive symptoms for FA group were the primary caregivers. These results may be related with the oversensitivity of the mothers about the symptoms in their infants actually not related with FA. Another possibility may be the psychological factors intrinsic to these mothers that may lead them to think that their children had FA (29).

The scores were also higher in non-IgE-mediated FA than those of IgE-mediated. This result may be related with the absence of a laboratory test to confirm non-IgE-mediated FA or diagnostic delays with the longer periods waiting in anxiety for the mothers. This may also be associated with the nature of symptoms. Seeing blood in stool may be very worrisome for a mother. In a study which found poorer physical QoL in non-IgE-mediated FA than that in IgE-mediated, the authors concluded that extra manifestations such as pain, hypermobility and fatigue and delays in diagnosis due to absence of laboratory tests may had 
an impact on the emotional psychological health of children (30). In another study, poor physical and emotional functioning associated with non-IgE-mediated FA was thought to be related with poor symptom recognition, delay in diagnosis and noxious symptoms such as sleep deprivation due to abdominal pain and feeding difficulties (31).

External stimuli affecting maternal feelings may affect the breastfeeding period. Nearly $50 \%$ of mothers reported that their breast milk had a change in color, volume, consistency and even more than two thirds of mothers in this study stated that their breast milk were decreased. Changes in breastmilk may also be due to extensive dietary eliminations. Almost half of True-FA group and 1/3 of False-FA group said to exclude $>20$ nutrients from their diets. Moreover, uncommon allergens such as vegetables and fruits were excluded by almost $20-30 \%$ of mothers. The ultimate allergens responsible for the reactions were actually just one type of food, for many it was only milk and not more than two in the majority of the cases. As thought from the perspective that relatively small number of allergens cause a high proportion of FA, it was obvious that mothers followed extensive unnecessary elimination diets (2). Extensive number of foods being avoided may also be a factor increasing maternal stress (32).

The evaluation of breastfeeding mothers with infants $<1$ year separately from older ages, comparison with a healthy control group, comparisons between Physician diagnosed FA vs Indecisive symptoms for FA, IgE- vs non-IgE-mediated FA using multiple standardized validated tools were the strengths of the study. The small number of patients within subgroups and lack of re-evaluation after remission of FA were the limitations. It is not well-known that the negative psychosocial functioning is caused by FA itself or by dietary elimination. Even though it was planned to include the cases with FA but without dietary elimination, this group is missing in the study due to the very few numbers of cases admitted to the study clinics.

In further studies, re-assessment of the mothers after remission of FA may answer the question of whether the psychosocial problems in mothers are pertinent to mothers themselves or related with burdensome aspects of FA.

In conclusion, breastfeeding mothers having children with FA were anxious, depressive and had many psychosocial symptoms which affected mother-to-infant bonding and interpersonal relationships. The Indecisive symptoms for FA and non-IgE-mediated FA groups had worse scores that the physician diagnosed FA and IgE-mediated FA groups, respectively.

Psychologic and social support and changes in the attitude of the physicians to the mothers who avoid major foods form their diet to care their infants in allergy clinics should be re-visited.

\section{References:}

1. Bock SA, Munoz-Furlong A, Sampson HA. Further fatalities caused by anaphylactic reactions to food, 2001-2006. J Allergy Clin Immunol 2007;119:1016-18.

2. Sampson HA, Aceves S, Bock SA, James J, Jones S, Lang D, et al. Food allergy: a practice parameter update-2014. J Allergy Clin Immunol 2014;134:1016-25.e43.

3. Cummings AJAJ, Knibb RC, King RM, Lucas JS. The psychosocial impact of food allergy and food hypersensitivity in children, adolescents and their families: a review. Allergy 2010;65(8):933-45.

4. Abdurrahman ZB, Kastner M, Wurman C, Harada L, Bantock L, Cruickshank H et al. Experiencing a first food allergic reaction: a survey of parent and caregiver perspectives Allergy Asthma Clin Immunol 2013;9(1):18.

5. Ferro MA, Van Lieshout RJ, Ohayon J, Scott JG. Emotional and behavioral problems in adolescents and young adults with food allergy. Allergy 2016;71:532-40.

6. Feng C, Kim JH. Beyond Avoidance: the Psychosocial Impact of Food Allergies. Clin Rev Allergy Immunol. 2019;57:74-82.

7. Reddy K, Kearns M, Alvarez-Arango S, Carrillo-Martin I, CuervoPardo N, Cuervo-Pardo L, et al. YouTube and food allergy: an appraisal of the educational quality of information. Pediatr Allergy Immunol 2018;29:410-6. 
8. Derogatis, LR, Cleary, PA. Confirmation of the dimensional structure of the SCL-90: A study in construct validation. Journal of clinical psychology 1977; 33:981-89.

9. Dağ İ. Belirti Tarama Listesi (Scl-90-R)'nin Üniversite Öğrencileri için güvenirliği ve geçerliği. Türk Psikiyatri Dergisi 1991;

10. Beck AT. An inventory for measuring depression. Arch Gen Psychiatry 1961;4:561-71.

11. Hisli N. Beck depresyon envanterinin universite ogrencileri icin gecerliligi, guvenilirligi.(A reliability and validity study of Beck Depression Inventory in a university student sample). J Psychol 1989;7:3-13.

12. Beck AT, Epstein N, Brown G, Steer RA. An inventory for measuring clinical anxiety: Psychometric properties. J Consult Clin Psychol 1998;56:893-97.

13. Ulusoy M, Sahin, NH, Erkmen H. The Beck anxiety inventory: psychometric properties. Journal of cognitive psychotherapy 1998;12:163-72.

14. Brockington IF, Oates J, George S, et al. A screening questionnaire for mother-infant bonding disorders. Arch Womens Ment Health 2001;3:133-40.

15. Brockington IF, Fraser C, Wilson D. The Postpartum Bonding Questionnaire: a validation. Arch Womens Ment Health 2006; 9:233-42.

16. Bowlby J. The nature of the child's tie to his mother. International journal of psycho-analysis 1958;39:350-73.

17. Akman İ, Kuscu MK, Yurdakul Z, Özdemir N, Solakoğlu M, Orhon L, et al. Breastfeeding duration and postpartum psychological adjustment: role of maternal attachment styles. Journal of paediatrics and child health 2008;44:369-73.

18. Herbert L, Shemesh E, Bender B. Clinical Management of Psychosocial Concerns Related to Food Allergy. J Allergy Clin Immunol Pract 2016;4:205-13.

19. Fedele DA, McQuaid EL, Faino A, Strand M, Cohen S, Robinson J, et al. Patterns of adaptation to children's food allergies. Allergy 2016;71:505-13.

20. Quigley J, Sanders GM. Food Allergy in Patients Seeking Mental Health Care: What the Practicing Psychiatrist Should Know. Curr Psychiatry Rep 2017;19:99.

21. Polloni L, Schiff S, Ferruzza E, Lazzarotto F, Bonaguro R, Toniolo A, et al.Food allergy and attitudes to close interpersonal relationships:An exploratory study on attachment. Pediatr Allergy Immunol 2017;28:458-63.

22. Williams NA, Hankey M: Support and negativity in interpersonal relationships impact caregivers'quality of life in pediatric food allergy. Qual Life Res 2015;24:1369-78.

23. King RM, Knibb RC, Hourihane JO. Impact of peanut allergy on quality of life, stress and anxiety in the family. Allergy 2009;64:461-88.

24. Warren CM, Gupta RS, Sohn MW, Oh EH, Lal N, Garfield CF, et al. Differences in empowerment and quality of life among parents of children with food allergy. Ann Allergy Asthma Immunol 2015;114:11725.

25. Lebovidge JS, Strauch H, Kalish LA, Schneider LC. Assessment of psychological distress among children and adolescents with food allergy. Allergy Clin Immunol. 2009;124:1282-8.

26. Rouf K, White L, Evans K. A qualitative investigation into the maternal experience of having young child with severe food allergy. Clin Child Psychol Psychiatry 2012;17:49-64.

27. Reichenberger J, Smyth JM, Kuppens P, Blechert J. "I will fast ... tomorrow": Intentions to restrict eating and actual restriction in daily life and their person-level predictors. Appetite. 2019 Apr 27;140:10-8. doi: 10.1016/j.appet.2019.04.019. [Epub ahead of print]

28. Birdi G, Cooke R, Knibb R. Quality of life, stress, and mental health in parents of children with parentally diagnosed food allergy compared to medically diagnosed and health controls. J Allergy (Cairo) 2016:1497375.

29. Knibb RC. Effectiveness of Cognitive Bahaviour Therapy for Mothers of Children with Food Allergy: A case series. Healthcare 2015;3:1194-211.

30. Foong RX, Meyer R, Godwin H, Dziubak R, Lozinsky AC, Reeve K, et al. Parental perception of their child's quality of life in children with non-immunoglobulin-E-mediated allergies. Pediatr Allergy Immunol 2017;28:251-6. 
31. Meyer R, Godwin H, Dziubak R, Panepinto JA, Foong RM, Bryon M, et al. The impact of life on families of children on an elimination diet for Non-immunoglobulin E mediated gastrointestinal food allergies. World Allergy Organ J 2017;10:8.

32. Sicherer SH, Noone SA, Munoz-Furlong A. The impact of childhood food allergy on quality of life. Ann Allergy Asthma Immunol 2001;87:461-4.

\section{Hosted file}

Tables-manuscript 13.04.2021.pdf available at https://authorea.com/users/411503/articles/ 520502-negative-effect-of-dietary-elimination-on-breastfeeding-mothers-having-infantswith-and-without-food-allergy 